\title{
Erratum to: Low Alpha-Fetoprotein Levels Are Associated with Improved Survival in Hepatocellular Carcinoma Patients with Portal Vein Thrombosis
}

\author{
Brian I. Carr ${ }^{1}$ Vito Guerra ${ }^{2}$
}

Published online: 26 February 2016

(C) Springer Science+Business Media New York 2016

\section{Erratum to: Dig Dis Sci}

DOI 10.1007/s10620-015-3922-3

The "Result" section in the abstract of this original publication of this manuscript contained an error.

The word "groups" present in the original manuscript was inadvertently deleted during the production process, thus changing the meaning of entire section. The correct result section of the abstract is given below:

Results We analyzed a large HCC cohort containing 366 (63.3\%) PVT-positive patients and found that PVT is associated with patients having larger tumors and higher levels of alpha-fetoprotein (AFP) and des-gamma carboxyprothrombin (DCP). We identified in patients with normal bilirubin levels $(\leq 2.0 \mathrm{mg} / \mathrm{dl})$, two PVT-positive patient groups, having higher and lower AFP levels, respectively. They differed in the significantly better prognosis of the low AFP patients, which may be useful for patient management decisions.

The online version of the original article can be found under doi: 10.1007/s10620-015-3922-3.

\footnotetext{
Brian I. Carr

brianicarr@hotmail.com

1 Izmir Biomedicine and Genome Center, Dokuz Eylul

University, Izmir, Turkey

2 IRCCS de Bellis, National Institute for Digestive Diseases, Castellana Grotte, Italy
} 